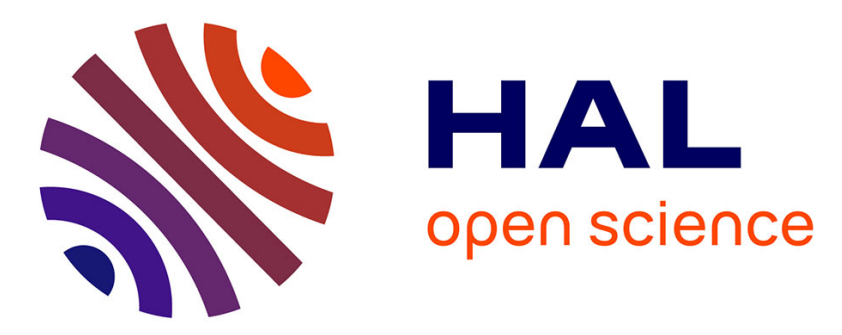

\title{
Features of spontaneous pointing gestures in toddlers
}

Hélène Cochet, Jacques Vauclair

\section{To cite this version:}

Hélène Cochet, Jacques Vauclair. Features of spontaneous pointing gestures in toddlers. Gesture, 2010, 10, pp.86-107. 10.1075/gest.10.1.05coc . halshs-01464524

\section{HAL Id: halshs-01464524 https://shs.hal.science/halshs-01464524}

Submitted on 30 Jan 2019

HAL is a multi-disciplinary open access archive for the deposit and dissemination of scientific research documents, whether they are published or not. The documents may come from teaching and research institutions in France or abroad, or from public or private research centers.
L'archive ouverte pluridisciplinaire HAL, est destinée au dépôt et à la diffusion de documents scientifiques de niveau recherche, publiés ou non, émanant des établissements d'enseignement et de recherche français ou étrangers, des laboratoires publics ou privés. 


\section{John Benjamins Publishing Company}

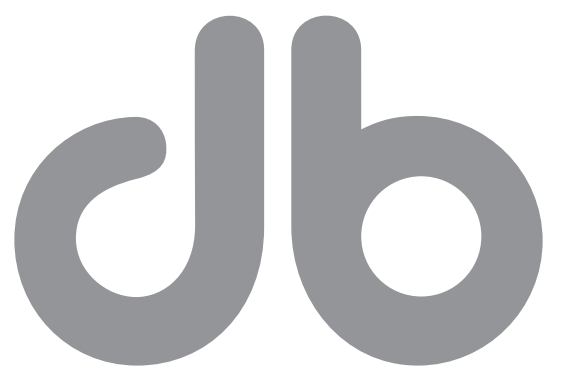

This is a contribution from Gesture 10:1

(C) 2010. John Benjamins Publishing Company

This electronic file may not be altered in any way.

The author(s) of this article is/are permitted to use this PDF file to generate printed copies to be used by way of offprints, for their personal use only.

Permission is granted by the publishers to post this file on a closed server which is accessible to members (students and staff) only of the author's/s' institute, it is not permitted to post this PDF on the open internet.

For any other use of this material prior written permission should be obtained from the publishers or through the Copyright Clearance Center (for USA: www.copyright.com). Please contact rights@benjamins.nl or consult our website: www.benjamins.com Tables of Contents, abstracts and guidelines are available at www.benjamins.com 


\title{
Features of spontaneous pointing gestures in toddlers
}

\author{
Hélène Cochet and Jacques Vauclair \\ Aix-Marseille University
}

\begin{abstract}
This study investigated the production of spontaneous pointing gestures in 26 toddlers, who were observed during free play time at day nursery. Pointing gestures and their different features (e.g., handedness, vocalizations, form and function of gesture) were recorded for a total observation time of 100 hours. Results revealed that the vast majority of pointing gestures were right-handed and accompanied by vocalizations, emphasizing the tight interconnection between speech and gesture from an early stage of development. Whole-hand gestures were more frequently used in imperative contexts, whereas index extensions were more frequently produced in declarative ones. Moreover, the use of declarative gestures and index extensions were found to increase with age. Implications concerning the origins of imperative and declarative pointing are discussed.
\end{abstract}

Keywords: spontaneous pointing gestures, toddlers, handedness, imperative vs. declarative function, index-finger vs. whole-hand extensions, gestural-vocal system

Several authors have highlighted the important role played by gesture in children's early development, reporting the existence of a significant relationship between communicative gestures produced around the end of the first year and the emergence of verbal skills at a later stage (e.g., Iverson \& Goldin-Meadow, 2005; Pizzuto \& Capobianco, 2005; Rowe \& Goldin-Meadow, 2009; Rowe, Özçaliskan, \& GoldinMeadow, 2008; Volterra, Caselli, Capirci, \& Pizzuto, 2005). The comprehension and production of communicative gestures involve cognitive processes that are essential for the acquisition of language: children develop some understanding of others' mental states through their ability to direct the attention of a recipient toward external events or objects (e.g., Liszkowski, Carpenter, Henning, Striano, \& Tomasello, 2004). These gestures are even regarded by some authors as a first step toward the emergence of a theory of mind (e.g., Camaioni, Perucchini, Bellagamba, 
\& Colonnesi, 2004). In the broad range of studies dedicated to the development of communicative skills, pointing gestures have been the subject of particular interest. Studies have focused either on the above-mentioned relationship between pointing gestures and language development (e.g., Butterworth \& Morissette, 1996), or on more global features associated with these gestures, such as the preference for the right hand (Bates, O'Connell, Vaid, Sledge, \& Oakes, 1986; Blake, O’Rourke, \& Borzellino, 1994; Vauclair \& Imbault, 2009; Young, Lock, \& Service, 1985) and different contexts of use (e.g., Tomasello, Carpenter, \& Liszkowski, 2007).

It is generally agreed that pointing is consistently accompanied by other behavioral expressions, especially vocalizations, which is regarded as one of the first signs of the tight coupling between speech and gesture. Speech-gesture links have been highlighted at both the behavioral and anatomical levels (e.g., Bates \& Dick, 2002; Gentilucci \& Dalla Volta, 2008; Iverson \& Thelen, 1999), leading to the hypothesis that communicative gestures are generated by a bimodal communication system in the left cerebral hemisphere, rather than by the system responsible for manipulative actions. This hypothesis implies greater activation of the left hemisphere when both modalities are simultaneously engaged, resulting in a greater degree of right-handedness. Investigations of manual activity during natural conversation in adults have indeed revealed a right-hand bias when the participants are speaking, though not when they are only listening (Kimura, 1973; Saucier \& Elias, 2001). The influence of vocal behavior on the degree of manual preference for communicative gestures has also been demonstrated in nonhuman primates: Hopkins and Cantero (2003) observed a greater degree of right-handedness in chimpanzees when food-begging gestures were produced along with vocalizations. The left cerebral hemisphere may thus be more highly activated when communicative gestures and vocalizations are produced simultaneously. One of our goals in the present study was to directly test this hypothesis. If the relationship between language and communicative gestures is established at a very early stage, we would expect pointing gestures in toddlers to be more right-handed when accompanied by vocalizations than gestures produced on their own.

In many developmental studies, pointing is defined as the extension of the arm and the index finger toward an object, person or event. However, this definition is regarded as too restrictive by some authors, for whom the essence of pointing lies in its function, namely the intentional attempt to direct someone's attention toward a referent. Wilkins (2003), for instance, has defined pointing as the use of some part of the body to make a deictic gestural reference, whether it is the hand, the mouth or the eyes. One definition of pointing gestures adopted by several authors includes both the index finger on its own and the full hand with all fingers extended (Brooks \& Meltzoff, 2008; Gullberg, de Bot, \& Volterra, 2008; Liszkowski, Carpenter, \& Tomasello, 2007, 2008). References to "whole-hand pointing" are 
more widespread in studies of nonhuman primates, where the traditional finger extension is less frequently observed than in human primates (Leavens, Hopkins, \& Bard, 1996; Leavens \& Hopkins, 1998).

This broader definition of pointing gestures brings up the issue of how to distinguish between pointing and another communicative gesture, usually referred to as a "request gesture". The latter is produced in order to obtain a desired object and is generally described as an extension of the arm toward the object, sometimes with a repeated opening and closing of the hand (Capirci, Contaldo, Caselli, \& Volterra, 2005; Gullberg et al., 2008). The fact that this repeated hand movement is not consistently observed for request gestures raises the question of whether there really is a difference between pointing toward an object with the whole hand in order to obtain that object and a requesting gesture. More confusing still, "request" is not the only term used to describe arm extensions toward an attractive object intended to make the adult give the child that object. Other terms found in the literature include "open-handed reaching" (Masur, 1983), "spread" (Fogel \& Hannan, 1985), "ritualized reaches" (Iverson \& Goldin-Meadow, 2005) and "reaching" (Franco \& Butterworth, 1996). The use of the word "reaching" can cause difficulties because it conveys different meanings: it primarily refers to the act of prehension (stretching out to grasp an object within one's reach), but can sometimes imply a communicative function. As stated above, children produce reaching gestures in order to obtain an out-of-reach object. Some authors have made a distinction between "reaching-in gestures" and "reaching-out gestures" (Blake et al., 1994). The former, which are similar to grasping, do not involve any communicative intention, unlike reaching-out gestures. In the study by Blake et al. (1994), the latter were right-handed in 8-and 12-month-old children, whereas reaching-in gestures were not. They were also accompanied by vocalizations more often than reachingin gestures. These results emphasize the communicative nature of "reach-outs", as well as their equivalence with request gestures. It would therefore be helpful for researchers to arrive at an agreement about the accurate definition of communicative gestures, first by disambiguating the use of "reaching gestures" and then, more generally, by using the same terms to refer to the same behaviors.

For this to happen, the functions of pointing must systematically be taken into account when studying gestural communication. Two main functions of pointing gestures have been described so far (Bates, Camaioni, \& Volterra, 1975; Camaioni, 1997). Imperative pointing is used by children to formulate a request, whereas the purpose of declarative pointing is to share an interest in an object or event with someone. The latter has recently been divided into "expressive" and "informative" declarative pointing (Tomasello et al., 2007). In the expressive subtype, the child seeks to share his or her enthusiasm with an adult about a common referent, while in the informative subtype, the child points to an object in order to help the adult, 
providing him/her with the information he/she needs. Tomasello et al. (2007) regard these gestures as subtypes of declarative pointing because they both rely on psychological processes that go well beyond the conception of the adult as a causal agent, as opposed to imperative pointing.

The fact that imperative and declarative pointing gestures are used in different contexts and for different purposes raises the question of their origin. Some gestures are derived from practical actions and acquire a communicative function via a process called "ontogenetic ritualization" (Tomasello \& Call, 1997). An action gradually becomes ritualized into a communicative signal through a partner's reaction to it. Imperative pointing, which is regarded as being equivalent to a request gesture, may originate from simple reaching actions. This abstraction from object-directed actions may account for similar structural characteristics, namely for the use of the whole hand. By contrast, declarative pointing gesture, as a means of sharing an attitude about a common referent with other individuals, may develop through social interactions and imitation processes. Several differences that have been observed between imperative and declarative pointing support the hypothesis of different origins for these gestures. Camaioni et al. (2004) assessed the understanding of adults' intentions by infants at the ages of 12 and 15 months through their ability to reproduce other people's intended acts after observing them fail to perform these acts. They found that the ability to understand intentions was linked to the production of declarative, but not imperative pointing. Moreover, declarative pointing emerged later than imperative pointing (e.g, Camaioni et al., 2004). A study has also shown that comment gestures, including declarative pointing, predict later communicative competence on the Peabody Picture Vocabulary Test (Dunn \& Dunn, 1981), whereas reach-request gestures produced at the beginning of the first year are negatively correlated to language measures at 3 years (Blake, Vitale, Osborne, \& Olshansky, 2005). Imperative function thus does not seem to be related to verbal communication. By contrast, declarative pointing in toddlers already reflects features of human language, namely social cognition and cooperation. As the main language functions are lateralized in the left cerebral hemisphere in the vast majority of people, investigating handedness for imperative and declarative pointing may shed light on the potentially different nature of these gestures. We may then observe different forms and degrees of right-handedness, depending on the function and origin of the pointing gestures. For instance, if imperative pointing is ritualized from a reaching action, we may observe more gestures produced with the whole hand, compared with declarative pointing. Gestures involving a request function may also be less righthanded than declarative ones.

The present study focused on several features of pointing gestures: handedness, form, function and the links between pointing and verbal behavior. Never 
before had all these aspects been studied together in humans, and we believed that recording observations was an efficient way of doing so. The aim of our investigation was thus to provide an overview of the entire range of forms and functions of pointing gestures produced by young children using naturalistic methods, that is through the observation of their spontaneous communicative gestures at daycare centers. Our first hypothesis was that we would find a right bias for communicative gestures, in line with several previous studies (e.g., Bates et al., 1986; Vauclair \& Cochet, submitted; Young et al., 1985). Our second hypothesis concerned the difference between the right bias of gestures produced on their own and the bias of gestures produced with vocalizations, the assumption being that the latter would be stronger than the former. Finally, we hypothesized that the pattern of pointing gestures would vary according to their intended function. We expected to observe differences in both the handedness and the form of the gestures, depending on their imperative or declarative function.

\section{Method}

\section{Participants}

The participants were 26 children ( 15 girls and 11 boys), observed at a daycare center. These children were divided into four groups which attended the daycare center on different moments (four different half days), including two groups of 7 ( 4 girls and 3 boys) and two groups of 6 ( 4 girls and 2 boys for the first one; 3 girls and 3 boys for the other one). Six female nursery staff members were always present with the children, interacting with and looking after them. Children were observed between 5 and 10 separate sessions depending on the group, over a three-month period. The sessions were each separated by at least one week and at most three weeks. Children were observed during three-hour blocks of time, in the morning or in the afternoon, resulting in a total observation time of approximately 100 hours. The mean number of sessions per child was 7.85 (S.D. $=2.4$ ), corresponding to a mean duration of 23.5 hours of recording (S.D. $=7.2$ ).

Children were aged between 11 months and 16 days and 37 months and 24 days on the first day of observation $(M=23.6 ;$ S.D. $=6.9)$. There was no significant difference in the mean number of sessions as a function of age, $F(2 ; 23)=0.74 ; n s$ (see Table 1). 
Table 1. Mean number of sessions depending on the age of the participants.

\begin{tabular}{llc}
\hline Age range & Mean number of sessions \pm S.D. & $\mathrm{N}$ \\
\hline $11.5-20$ months & $8.36 \pm 2.3$ & 11 \\
$20-29$ months & $7 \pm 2.6$ & 8 \\
$29-38$ months & $8 \pm 2.5$ & 7 \\
\hline
\end{tabular}

\section{Procedure}

The observations were conducted during free play times and included a snack time. In order not to interfere with the different activities and interactions, the observer always remained on the periphery of the group. As this study focused on pointing gestures, data were collected using a behavior-dependent sampling method. The small size of the groups being observed allowed the experimenter to record all the communicative pointing gestures accurately and efficiently on a datasheet, as and when they occurred. The observer was highly trained to record infants' gestural behaviors and she had spent a few days in the day-care center before starting the data collection, in order to observe the interactions between children.

Pointing gestures were defined as the extension of the arm towards a referent (object or event) involving a clear communicative intention through gaze, vocalization or other clear evidence of an effort to direct someone's attention. Several features characterizing pointing gestures were then taken into account. The observer (HC) first noted whether the gesture was produced with the index finger extended and the other fingers curled or with a less conventional form and all fingers extended ("whole-hand" pointing, Leavens \& Hopkins, 1999). For each gesture, the observer recorded which hand was used and whether or not the gesture was accompanied by a vocalization. Vocalizations consisted of either words or other vocal communicative productions (e.g., pseudowords or speech sounds). Whining and crying were not included. Vocalizations were considered to be produced co-temporally with a gesture when the two events occurred simultaneously or within a two-second interval. The observer also noted whether or not the pointing gesture was coordinated with gaze alternation, that is, whether children shifted their gaze between the target and the social partner while pointing or within a two-second interval after the production of the gesture.

Each pointing gesture was also classified according to its function. When the child pointed to an out-of-reach object so that the adult gave him/her or did something with it that the child could not do by him/herself, it was coded as an imperative pointing. When the child sought to direct a recipient's attention toward a referent in order to share interest in it or provide the recipient with helpful information, the gesture was coded as a declarative pointing. In some cases, when 
the child's intention was not obvious at first sight, the observer relied on the adult's behavioral reaction to the child's pointing and on the child's behavior following the adult's first reaction. For example, a gesture was classified as imperative if the child showed signs of satisfaction and ceased the gesture after the adult gave him/ her the object pointed at, and as declarative if the gesture ceased after the adult commented on the referent (see Carpenter, Nagell, \& Tomasello, 1998).

Within the more general category of declarative behaviors, we initially distinguished between the informative function (e.g., when the child helps the adult by pointing to an object he/she is looking for) and the expressive function (e.g., when the child wants the adult to see an event of interest and share enthusiasm about it) (Tomasello et al., 2007). However, as fewer than five informative pointing gestures were produced overall, we decided to group these two functions into a single category.

In order to avoid any effects of positional bias on hand use, gestures were only recorded when (1) the child was in a symmetrical posture (the body was in a straight position and both arms were at an equivalent distance from the body) with both hands initially free, and (2) the referent was positioned centrally in front of the gesturer. Even when several individuals meeting these two criteria were pointing at the same time (something which happened very rarely), the observer was still able to score the pointing behaviors of two individuals simultaneously. If more than two children were pointing simultaneously, instead of trying to record all the gestures, the experimenter chose two of them in order to maintain a comparable number of observations for each participant: if the number of data points previously recorded for one individual was already high, this individual child's pointing was not recorded. However, this only happened three times during the 100 hours of observation.

The sessions were videotaped for two groups in order to evaluate interobserver reliability (the camera was placed in a corner of the room). We were not allowed to film the two other groups because some parents did not give their agreements. Analyses of variance did not reveal any difference between groups for any variables.

\section{Reliability}

Reliability was assessed on a subset of the videotaped sessions (approximately 15h) by an independent coder who was blind to the hypotheses of the study. The data obtained by the first observer from sheet recording were compared to the data recorded by the second observer from videotapes. First, 63 gestures produced by 12 children (12.5\% of the total number of gestures) were recognized as communicative by both coders (inter-observer agreement was 100\%). Within these 63 
gestures, the analyses revealed high to very high inter-observer reliability. Cohen's kappa statistics for coding decisions were 1 for the hand used, .85 for the function of the pointing gesture, .71 for handshapes, .82 for vocalizations and .65 for gaze alternation.

A few gestures could not be taken into account for the assessment of reliability when the video was not perfectly centered on the child's gesture (which could not be avoided as there was only one camera in the room), or for example, when an adult passed in front of the camera. Nevertheless, as the high agreement between coders highlighted the reliability of the data recorded by the observer, these gestures were included in the analyses.

\section{Data analysis}

The data were summed across the test sessions. An individual handedness index score (HI) was calculated for each child using the formula $(\mathrm{R}-\mathrm{L}) /(\mathrm{R}+\mathrm{L})$, where $\mathrm{R}$ and $\mathrm{L}$ stand for the total right- and left-hand responses. The $\mathrm{HI}$ values lay along a continuum from -1 to 1 , with the $+/-$ sign indicating hand-preference direction and the absolute value reflecting hand-preference strength. Handedness indices were calculated for pointing gestures and analyzed with respect to their form (index-finger vs. whole-hand pointing), function (declarative vs. imperative pointing) and vocalizations (gestures that were accompanied by vocalizations vs. those that were not). All analyses were performed using parametric statistics with alpha set to $p<0.05$.

\section{Results}

\section{Hand preference for pointing}

A total of 503 gestures were collected ( $93.4 \%$ of which were addressed to an adult) and the number of observations per participant varied from 5 to $63(M=19.3$; S.D. $=15.1)$. As expected, we observed a significant right-hand bias for communicative gestures, as 428 gestures $(85.1 \%)$ were right-handed and $75(14.9 \%)$ were left-handed. The mean numbers of right-handed and left-handed gestures produced were $16.5($ S.D. $=13.8)$ and $2.9($ S.D. $=3.1)$, respectively $(t(26)=4.91$; $p<.001)$. Handedness scores varied between 0.11 and 1 and the mean handedness index was $0.68(S . D .=0.25)$. 


\section{Gaze alternation}

Each of the 26 children exhibited gaze alternation between the object of the pointing gesture and the face of the recipient. Gaze alternation was observed in $56.5 \%$ of cases. There was no significant difference between the mean number of gestures accompanied by gaze alternation $(M=10.9 ; S . D .=9.8)$ and the mean number of gestures produced without gaze alternation $(M=8.4 ; S . D .=7.2, t(26)=1.05 ; n s)$.

\section{Accompanying vocalizations}

A total of 435 pointing gestures were accompanied by a vocalization ( $86.5 \%)$ and $68(13.5 \%)$ were not. Children's gestures were significantly more frequently accompanied by vocalizations $(M=16.7 ; S . D .=13.7)$ than produced on their own $(M=2.6 ; S . D .=2.9 ; t(18)=5.16 ; p<.001)$. To test our second prediction, we assessed whether the right-hand bias for pointing gestures was stronger when these gestures were accompanied by vocalizations. Eight of the 26 children only produced gestures accompanied by vocalizations, so they were excluded from the analysis. No significant difference was observed between $\mathrm{HI}$ for pointing accompanied by vocalizations (vocal HI) and HI for unaccompanied pointing (non-vocal HI) $(t(18)=0.067 ; n s)$. Our results therefore did not confirm our initial hypothesis. It should, however, be noted that because of the small number of pointing gestures produced without any vocalization $(M=2.6, S . D .=2.9)$, the handedness scores associated with these gestures may not have been entirely representative of the children's degree of handedness. The comparison between vocal HI and nonvocal $\mathrm{HI}$ should thus be interpreted with some caution.

\section{Form and function of pointing gestures}

Every child produced pointing gestures with the index finger extended and 20 of the 26 participants also produced whole-hand pointings. Four hundreds and fourteen pointing gestures $(82.3 \%)$ were characterized by the conventional extension of the index finger, whereas 89 gestures (17.7\%) were produced with the whole hand. Children's gestures were significantly more frequently produced with the index extended $(M=15.9 ;$ S.D. $=14.2)$ than with the whole hand $(M=3.4 ; S . D .=4.0$; $t(20)=4.33 ; p<.001)$.

Regarding the communicative intent of the pointing gestures, 160 gestures (31.8 \%) had an imperative function and 343 gestures (68.2 \%) a declarative one, be it sharing an interest in an object or event or providing helpful information to the recipient. Four children used pointing gestures only in the declarative context. The mean number of declarative gestures $(M=13.2$; S.D. $=12.2)$ was significantly 
greater than the mean number of imperative gestures $(M=6.2 ; S . D .=6.4$; $t(22)=2.60 ; p<.05)$.

On average, $94.1 \%$ of declarative pointing gestures were produced with the extended index finger vs. $53.9 \%$ of imperative pointing gestures. This difference was significant $(t(22)=5.61 ; p<.001)$.

We first investigated which handshape more frequently characterized imperative and declarative functions, and then, reciprocally, we examined which function index-finger and whole-hand pointing were more frequently used for. As far as declarative pointing is concerned, the mean number of gestures produced with the index finger was higher than the mean number of whole-hand gestures $(t(26)=5.02 ; p<.001)$. This difference was observed for both right-handed $(t(26)=4.70 ; p<.001)$ and left-handed pointing $(t(26)=3.66 ; p<.001)$. As far as imperative pointing is concerned, there were no significant differences in the mean number of gestures produced as a function of handshapes $(t(26)=0.37 ; n s)$, either for right-handed gestures $(t(26)=0.44 ; n s)$ or for left-handed ones $(t(26)=-0.47$; $n s)$. The mean proportions of declarative and imperative pointing gestures produced according to gesture form are shown in Figure 1.

Index-finger pointings were more frequently used with a declarative function than with an imperative one $(t(26)=4.14 ; p<.001)$. Whole-hand gestures, on the contrary, were more frequently produced with an imperative function $(t(26)=3.86$; $p<.001)$. This was true for both right-handed $(t(26)=-3.40 ; p<.01$ for index-

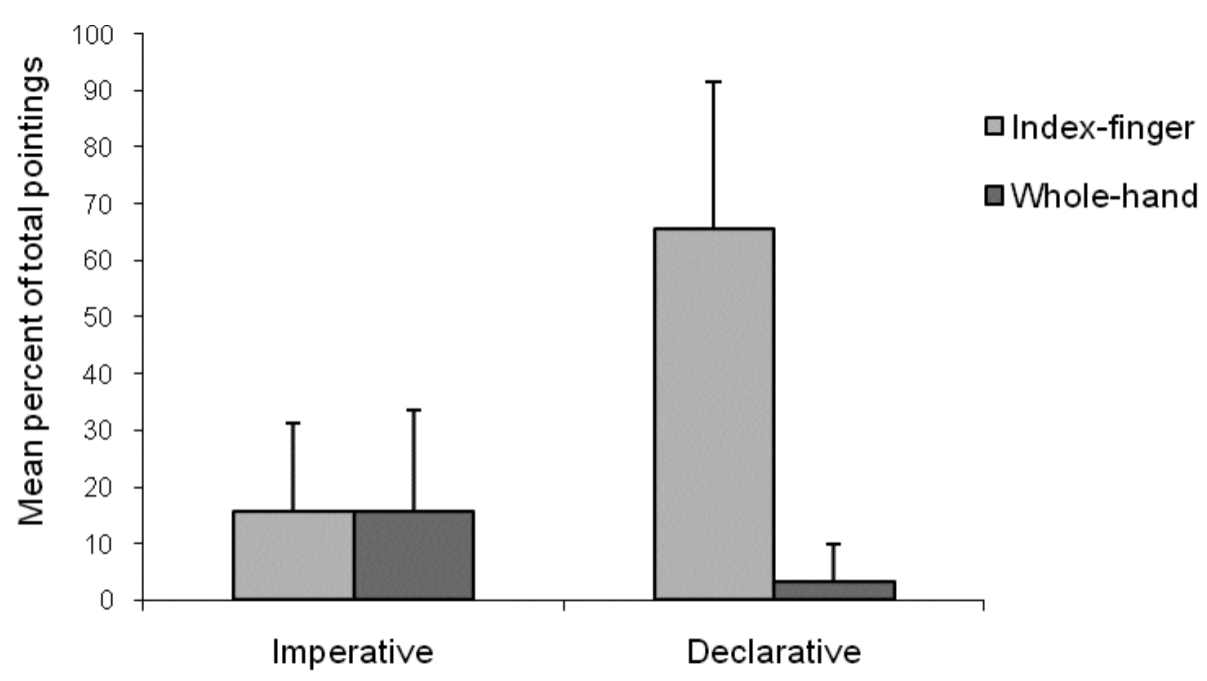

\section{Function of the pointing gesture}

Figure 1. Mean proportions of declarative and imperative pointing gestures according to gesture form. 
finger gestures and $t(26)=3.35 ; p<.01$ for whole-hand gestures) and left-handed gestures $(t(26)=-2.88 ; p<.01$ for index-finger gestures and $t(26)=2.43 ; p<.05$ for whole-hand gestures).

The HIs for declarative and imperative pointing were compared using Student's t-test. Mean HIs $(0.74 \pm 0.24$ and $0.75 \pm 0.32$, respectively) did not differ $(t(22)=-0.11 ; n s)$. We also compared the HIs for index-finger pointing and wholehand pointing, but there was no difference either in the degree of the right-hand bias $(t(20)=-0.81 ; n s)$.

Our third hypothesis was thus partially confirmed, insofar as the form of the pointing gestures, though not their handedness pattern, varied according to the function of the gesture. Moreover, another feature appeared to differ between imperative and declarative pointing: the proportions of imperative and declarative gestures produced simultaneously with vocalizations were respectively 58.8\% (S.D. $=35.6)$ and 91.7\% (S.D. =14.7). Declarative gestures were thus more frequently accompanied by vocalizations than imperative gestures $(t(22)=-4.00$; $p<.001)$. Regarding visual behavior, there was no difference between the two types of pointing in the mean proportions of gestures accompanied by gaze alternation $(t(22)=0.08 ; n s)$.

\section{Gender}

There was no significant difference in pointing behavior according to gender, either in the total number of gestures produced $(t(26)=-0.95 ; n s)$, the handedness index associated with pointing $(t(26)=-1.72 ; n s)$ or the function of the gestures $(t(26)=0.38 ; n s)$.

Age

There was no significant relationship neither between the age of the participants and the total number of gestures they produced $(r=-0.24 ; n s)$, nor between age and the degree of right-handedness associated with pointing $(r=0.24 ; n s)$. The right-hand bias did not become stronger with age. Moreover, the proportion of gestures produced with accompanying vocalizations did not increase as a function of age $(r=0.31 ; n s)$. But we observed significant relationships between age and function of pointing gesture $(r=0.55 ; p<.01)$ and between age and form of gesture $(r=0.53 ; p<.01)$. As illustrated in Figure 2 , when children grew older, they produced an increasing number of pointing gestures with a declarative function and with the index finger extended. 


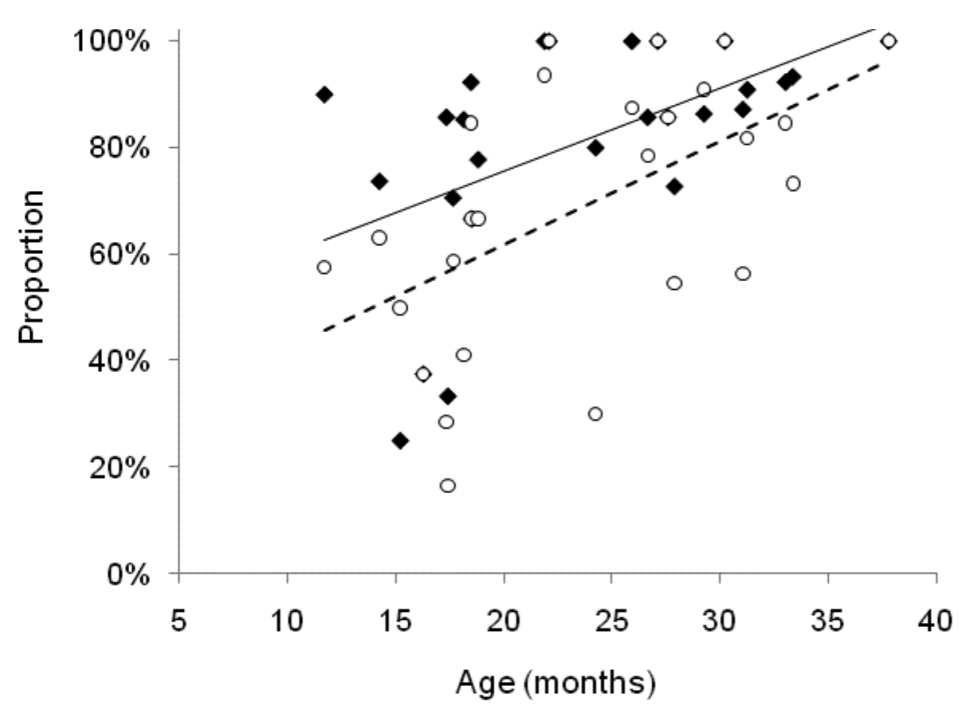

\section{- Proportion of index-finger extensions $\circ$ Proportion of declarative gestures}

Figure 2. Relationship between age, proportion of index-finger extensions (solid line) and proportion of declarative gestures (dashed line).

\section{Discussion}

This research was designed to probe the features of spontaneous pointing gestures in young children. To our knowledge, it was the first study to investigate the characteristics of pointing gestures under naturalistic conditions in a day care center. The observation of these spontaneous gestures may shed new light on the development of communicative behavior in toddlers. The results showed a strong and significant right-hand preference for pointing gestures, in line with previously reported findings (Bates et al., 1986; Blake et al., 1994; Young et al., 1985). The mean Handedness Index (HI) observed in the present study (0.68) was stronger than the mean HI for pointing reported by Vauclair and Imbault (2009) in experimental conditions (0.52). This difference may simply be due to the absence of left-handed participants in the present study (all the HI were positive), which was not the case in the study by Vauclair and Imbault (2009), as they recruited a larger sample of participants.

The right-handed bias did not increase as children grew older, that is, between approximately 1 and 3 years of age (the youngest participant was aged 11 months and 16 days at the beginning of the observations and the oldest one was 37 months and 24 days). A study of toddlers aged 13-28 months (Bates et al.., 1986) also failed to reveal any strengthening of the right-sided asymmetry over this period. An increase in the right-sided bias for pointing gestures had previously been reported, 
but it concerned lower age ranges than those selected for the present study (Blake et al., 1994; Young et al., 1985), indicating that the increasing involvement of the left cerebral hemisphere in the production of communicative gestures may take place even before children reached one year of age.

Communicative signals were expressed simultaneously in the gestural and vocal modalities in $86.5 \%$ of cases. This widespread use of vocalizations contrasts with results obtained with nonhuman primates, showing that the majority of gestures are not accompanied by vocalizations (Hopkins \& Cantero, 2003). The specificity of human communicative behavior, which lies in the vocal modality, therefore appears at a very early stage in development. Furthermore, it is interesting to note that the recipients of referential gestures produced by children were adults in the majority of cases. This result suggests that communicative skills may develop through interactions with adult caregivers and not with other children, in line with previous studies emphasizing the influence of adults' inputs (e.g., Kishimoto, Shizawa, Yasuda, Hinobayashi, \& Minami, 2007).

We did not observe any difference in the degree of right bias between gestures produced alone and gestures produced simultaneously with vocalizations, apparently disproving our hypothesis that a greater demand is placed on left hemisphere resources when both modalities are involved simultaneously. However, this finding may be explained by the relatively low number of gestures produced without vocalizations (the mean number of pointings produced on their own was 2.6 and eight children did not produce any at all). Consequently, the comparison of handedness scores for gestures produced with and without vocalizations was not based on an equivalent number of gestures. Moreover, no attempt was made in the present study to characterize the vocalizations and words produced by the children. It would be useful to study in a future research the different features of these communicative signals in greater depth, possibly using spectrographic analysis. A sound spectrograph would provide measures of the tone, rhythm, amplitude and frequency of vocal sounds, which could help to distinguish between different vocalizations.

A significant relationship was observed between the form and function of pointing gestures. Index extensions were more frequently used with a declarative function, whereas whole-hand gestures were more frequently produced with an imperative function. Moreover, in the vast majority of cases, declarative pointing was produced with the index finger, whereas imperative pointing was characterized equally by index-finger and whole-hand extensions.

These different structural characteristics, as well as the different motivational backgrounds of imperative and declarative pointing gestures, may be related to their different origins. Imperative gestures were more frequently associated with whole-hand extensions, which superficially resemble acts of prehension, than 
declarative gestures were. It may thus be hypothesized that imperative pointing substitutes for reaching actions by a process of ontogenetic ritualization (Tomasello \& Call, 1997). A behavior that is not initially a communicative signal becomes one through reciprocal social interactions: the child learns over repeated instances that his/her gesture elicits a particular action from the adult (in this case, the action of giving the child the desired object). Vygotsky (1988) had previously argued that pointing develops out of reaching, but this hypothesis only seems consistent with the imperative function of pointing.

Declarative pointing, by contrast, would not appear to emerge from a ritualization process, as almost all declarative pointing gestures were produced with the extended index. It has been suggested that index-finger pointing emerges not from a less differentiated form, but from the non-communicative finger extensions observed in infants from three months onwards (Butterworth, 2003; Masataka, 2003). One argument advanced to support this assumption concerns the changes that take place in the gestural repertoire in the course of early development: the frequency of index-finger extensions increases between 3 and 11/12 months, then decreases, whereas the frequency of index-finger pointing gestures starts to increase (Masataka, 2003). For this reason, among others, index-finger pointing is sometimes viewed as the basic and natural form of reference, which develops spontaneously (Butterworth, 2003). However, investigations of deictic behaviors in different cultures have revealed variations in the form of pointing gestures, indicating that index-finger pointing is not the universally preferred referential strategy. In some cultures, for example, lip-pointing is dominant and forms of manual pointing that are never or rarely encountered in some cultures are frequently observed in others, such as when the middle finger, not the index finger, is pointed toward a target (Kendon \& Versante, 2003; Wilkins, 2003).

These observations suggest that the use of the index finger for pointing is not universal and is, at least to some degree, socially transmitted to the infant. The development of index-finger extensions in the present study may indeed have involved an imitation process. When adults used pointing gestures to communicate with children, they did so with a declarative motive rather than an imperative one, and with the extended index. They sought to direct the child's attention toward a referent in order to share interest in it, for example when looking at pictures in a book. Fewer, if any, examples come to mind of a parent pointing with the whole hand toward an object so that the child will bring it to him/her. Studies should be conducted to investigate the function of pointing gestures produced by caregivers when interacting with children in order to help determine whether imitation is the major learning process at work in the development of declarative pointing.

We can thus raise the hypothesis that imperative and declarative pointing gestures develop in parallel and independently. Different developmental sequences 
in the emergence of imperative and declarative pointing have previously been reported: declarative pointing develops later than imperative pointing (e.g., Camaioni et al., 2004). Moreover, authors have shown that children with autism fail to understand and produce declarative pointing, but not imperative pointing (Camaioni, 1997). This hypothesis is also reinforced by the relationship reported in the present study between age and the function of the gesture. As children grow older, they use more and more pointing gestures in declarative contexts and with the index finger extended.

This increasing use of index-finger pointing may reflect a developmental tendency toward more symbolic forms of communication (e.g., Franco \& Butterworth, 1996). This hypothesis is supported by studies of nonhuman primates, as language-trained chimpanzees point more frequently with their index fingers than chimpanzees which have not experienced close relationships with humans (Leavens \& Hopkins, 1999). The extension of the index is then particularly likely to be observed in the context of close relationships with humans. Note that this is a favorable context for imitation to occur, but so far, this assumption has not been confirmed by empirical evidence, as some studies have reported that chimpanzees do not imitate (see Tomasello, 2006).

Even if the majority of whole-hand pointings were used in imperative contexts, imperative pointing gestures were produced both with the whole hand and with the index finger. Therefore, our initial proposal that imperative pointing and "request gestures" (e.g., Capirci et al., 2005) are identical communicative signals proved not to be entirely satisfactory. These gestures may share the same function, but their respective forms are somewhat different. As a few studies have observed a decrease in the production of reaching gestures (described as communicative and imperative gestures with all fingers extended) as children grow older (e.g., Blake, McConnell, Horton, \& Benson, 1992), we can assume that early imperative pointings are produced with the whole hand and later on with the extended index. This progressive shift in handshapes might be related to a progressive shift in the cognitive processes associated with imperative gestures. Tomasello and colleagues (2007) suggested that imperative motives form a continuum from ordering to suggesting. It could then be hypothesized that at an early stage, children understand the adult as a causal agent from whom they can get what they want, and later on, while imperative pointing becomes more frequently produced with the index-finger, the adult is regarded as an intentional agent who can decide to help the children. This perspective entails the possibility that the production of imperative pointing is different between non human primates and human children, that is, is related to different cognitive and social skills. In line with this hypothesis, it has been shown that human infants were able to request absent objects, whereas chimpanzees did not possess this ability (Liszkowski, Schäfer, Carpenter, \& Tomasello, 2009). 
Moreover, as the declarative gestures were almost exclusively characterized by index finger extension in the present study, we can also hypothesize that declarative pointing influences the structural characteristic of all deictic behaviors. Once index-finger pointing appears in the child's gestural communication system, imperative pointing behavior may gradually be modified to feature the index extension. Note that this does not exclude the possibility that imperative pointing may originate from non-communicative actions, or the idea that imperative and declarative pointing are functionally distinct.

More globally, the relationship between handshapes, functions and origins of pointing gestures is a complex issue, and as suggested by Tomasello (2006), some infants may learn to use pointing in one way and some in the other way. Even if the results of the present study showed some predominant developmental patterns in the production of pointing, there might be different developmental trajectories. For example, approximately $6 \%$ of declarative pointing gestures were produced with the whole-hand, by children who also produced declarative indexfinger points. Given the purpose of declarative pointing, it is unlikely that these whole-hand gestures emerge from prehension, but it remains very difficult to find out, empirically, whether pointing gestures are ritualized from reaching actions or learned though imitative process.

The distinction between imperative and declarative pointing was expected to encompass different patterns of handedness, reflecting different degrees of involvement of the left cerebral hemisphere. Imperative pointing, at least in the early stages, relies solely on the representation of people as causal agents, whereas declarative pointing implies the ability to represent and influence another person's attentional state, which is a crucial step in communication (Camaioni et al., 2004). Declarative pointing was thus expected to be more right-handed than imperative pointing, but in the event we failed to observe any handedness differences between the two types of gestures. Unless the difference in activation levels is too subtle to be reflected in manual preferences, imperative and declarative points may involve the left cerebral hemisphere to an equal extent, insofar as they are both communicative gestures. Moreover, some researchers do not support a cognitive distinction between imperative and declarative gestures. On the one hand, both types of gestures are regarded as instrumental acts that do not involve the understanding of others' attention (e.g., Moore \& Corkum, 1994), and, on the other hand, both imperative and declarative gestures would reveal an early form of psychological understanding (e.g., Liszkowski, 2005). This latter perspective has recently been supported by empirical findings (e.g., Liszkowski et al., 2009).

However, even if imperative gestures may not be related to simpler cognitive processes than declarative gestures, a clear distinction between both types of gestures in the present study relied on the incidence of accompanying vocalizations. 
Declarative gestures were more likely to be produced with vocalizations compared to imperative gestures, which emphasizes the close relation between declarative gestures and the vocal system. This result is important insofar as it may reflect different roles played by imperative and declarative pointing in language development. It would then be useful for future studies to measure children's language levels, in order to investigate whether language abilities are more strongly correlated with declarative than with imperative gestures.

Within declarative pointing, some authors have distinguished between the expressive and the informative function (e.g., Liszkowski, Carpenter, Striano, \& Tomasello, 2006; Pika, 2008). The expressive function, the one examined in our study, refers to the intention of sharing an interest with a communicative partner about a referent, whereas the purpose of informative pointing is to provide the other person with information he/she needs. For example, if we see that another person has mislaid an object, and we know where it is, we will point in its direction to help that person. This gesture, which is within the capability of 12-month-olds, involves an understanding of others as people with intentional and informational states. Informative pointing has been studied within experimental contexts, where artificial situations have been set up to elicit this gesture (Liszkowski et al., 2006). For instance, the experimenter, without apparently noticing, accidentally drops an object on the floor and then starts looking for it. The child is then likely to point toward the object. In the present study, very few instances of informative pointing were observed. Opportunities for a child to provide an adult with useful information were probably few and far between, but we can also assume that this gesture is not willingly produced outside a standardized context. Even if experimental studies of informative pointing are interesting from both a cognitive and a motivational point of view, it is noteworthy that this gesture is not really part of toddlers' spontaneous gestures.

All 26 children exhibited gaze alternation between the object of the pointing and the face of the recipient, but pointing gestures were not always associated with this behavior. Gaze alternation is usually regarded as a hallmark of intentional communication (e.g., Bates et al., 1975). The fact that gaze alternation did not consistently accompany pointing gestures in our study could therefore call into question the nature of the pointing gestures we recorded. Nevertheless, several factors need to be taken into account when deciding whether a communicative gesture is intentional and infants' gaze alternation may be influenced by many factors (Liszkowski, Albrecht, Carpenter, \& Tomasello, 2008). In the present study, the respective positions of the gesturer, recipient and referent of the pointing did not necessarily allow the child to alternate his/her gaze between the object and the adult. For example, if the child was seated on the adult's lap when pointing to a referent in front of him/her, gaze alternation was probably not observed because the 
child would have had to have turned round to see the adult's face. More generally, gaze alternation was less likely to occur when the adult and child were looking in the same direction. It was then easier for the child to direct the adult's attention to a referent and he/she probably felt less inclined to check the efficiency of his/her gesture via gaze alternation. Thus, pointing cannot be classified as communicative or non-communicative simply on the basis of visual orienting behavior (e.g., Liszkowski et al., 2008; Murphy, 1978). Future studies therefore need to focus more carefully on the situations in which the pointing gesture is produced. In a study comparing the declarative and requestive functions of communicative gestures, visual checking was found to be more closely associated with the declarative function of pointing (Franco \& Butterworth, 1996). This result was not observed in the present study, but Franco and Butterworth (1996) investigated visual behavior in experimental conditions that allowed the children to adopt standardized postures. As a consequence, they were not concerned with the different positions of the communicative partners and the extent to which they would favor gaze alternation.

Taken together, our results emphasize the relevance of distinguishing between imperative and declarative functions of pointing when investigating the development of communicative gestures in a natural setting. The results of the present observational study need to be investigated in experimental studies, where specific situations would elicit imperative and declarative pointing gestures. Standardized contexts would provide a fruitful comparison with our study regarding handedness, form and function of pointing gestures.

\section{Acknowledgements}

This research was supported by a French National Research Agency (ANR) grant reference ANR-08-BLAN-0011_01.

\section{References}

Bates, Elizabeth, Luigia Camaioni, \& Virginia Volterra (1975). The acquisition of performatives prior to speech. Merrill-Palmer Quarterly, 21, 205-226.

Bates, Elizabeth \& Frederic Dick (2002). Language, gesture and the developing brain. Developmental Psychobiology, 40, 293-310.

Bates, Elizabeth, Barbara O'Connell, Jyotsna Vaid, Paul Sledge, \& Lisa Oakes (1986). Language and hand preference in early development. Developmental Neuropsychology, 2, 1-15.

Blake, Joanna, Sheilah McConnell, Gayle Horton, \& Nancy Benson (1992). The gestural repertoire and its evolution over the second year. Early Development and Parenting, 1, 127-136. 
Blake, Joanna, Paula O'Rourke, \& Grace Borzellino (1994). Form and function in the development of pointing and reaching gestures. Infant Behavior and Development, 17, 195-203.

Blake, Joanna, Grace Vitale, Patricia Osborne, \& Esther Olshansky (2005). A cross-cultural comparison of communicative gestures in human infants during the transition to language. Gesture, 5, 201-217.

Butterworth, George (2003). Pointing is the royal road to language for babies. In Sotaro Kita (Ed.), Pointing: where language, culture, and cognition meet (pp.9-34). Mahwah, NJ: Lawrence Erlbaum Associates.

Butterworth, George \& Paul Morissette (1996). Onset of pointing and the acquisition of language in infancy. Journal of Reproductive and Infant Psychology, 14, 219-231.

Brooks, Rechele \& Andrew N. Meltzoff (2008). Infant gaze following and pointing predict accelerated vocabulary growth through two years of age: A longitudinal, growth curve modeling study. Journal of Child Language, 35, 207-220.

Camaioni, Luigia (1997). The emergence of intentional communication in ontogeny, phylogeny and pathology. European Psychologist, 2, 216-225.

Camaioni, Luigia, Paola Perucchini, Francesca Bellagamba, \& Cristina Colonnesi (2004). The role of declarative pointing in developing a theory of mind. Infancy, 5, 291-308.

Capirci, Olga, Annarita Contaldo, Cristina M. Caselli, \& Virginia Volterra (2005). From action to language through gesture: A longitudinal perspective. Gesture, 5, 155-177.

Carpenter, Malinda, Katherine Nagell, \& Michael Tomasello (1998). Social cognition, joint attention, and communicative competence from 9 to 15 months of age. Monographs of the Society of Research in Child Development, 255 (63), 1-143.

Dunn, Llyod M. \& Leota M. Dunn (1981). The Peabody Picture Vocabulary Test. Circle Pines, $\mathrm{MN}$ : American Guidance Service.

Fogel, Alan \& Thomas E. Hannan (1985). Manual acts of 9 to 15 week-old human infants during face-to-face interaction with their mothers. Child Development, 56, 1271-1279.

Franco, Fabia \& George Butterworth (1996). Pointing and social awareness: Declaring and requesting in the second year. Journal of Child Language, 23, 307-336.

Gentilucci, Maurizio \& Riccardo Dalla Volta (2008). Spoken language and arm gestures are controlled by the same motor control system. The Quarterly Journal of Experimental Psychology, 61, 944-957.

Gullberg, Marianne, Kees de Bot, \& Virginia Volterra (2008). Gestures and some key issues in the study of language development. Gesture, 8, 149-179.

Hopkins, William D. \& Monica Cantero (2003). From hand to mouth in the evolution of language: The influence of vocal behaviour on lateralized hand use in manual gestures by chimpanzees. Developmental Science, 6, 55-61.

Iverson, Jana M. \& Susan Goldin-Meadow (2005). Gesture paves the way for language development. Psychological Science, 16, 367-371.

Iverson, Jana M \& Esther Thelen (1999). Hand, mouth and brain: The dynamic emergence of speech and gesture. Journal of Consciousness Studies, 6, 19-40.

Kendon, Adam \& Laura Versante (2003). Pointing by hand in Neapolitan. In Sotaro Kita (Ed.), Pointing: where language, culture, and cognition meet (pp. 109-137). Mahwah, NJ: Lawrence Erlbaum Associates.

Kimura, Doreen (1973). Manual activity during speaking: I. Right-handers. Neuropsychologia, $11,45-50$. 
Kishimoto, Takeshi, Yasuhiro Shizawa, Jun Yasuda, Toshihiko Hinobayashi, \& Tetsuhiro Minami (2007). Do pointing gestures by infants provoke comments from adults? Infant Behavior and Development, 30, 562-567.

Leavens, David A. \& William D. Hopkins (1998). Intentional communication by chimpanzees: A cross-sectional study of the use of referential gestures. Developmental Psychology, 34, 813-822.

Leavens, David A. \& William D. Hopkins (1999). The whole-hand point: The structure and function of pointing from a comparative perspective. Journal of Comparative Psychology, $113,417-425$.

Leavens, David A., William D. Hopkins, \& Kim A. Bard (1996). Indexical and referential pointing in chimpanzees (Pan troglodytes). Journal of Comparative Psychology, 110, 346-353.

Liszkowski, Ulf (2005). Human twelve-month-olds point cooperatively to share interest with and helpfully provide information for a communicative partner. Gesture, 5 (1/2), 135-154.

Liszkowski, Ulf, Konstanze Albrecht, Malinda Carpenter, \& Michael Tomasello (2008). Infants' visual and auditory communication when a partner is or is not visually attending. Infant Behavior and Development, 31 (2), 157-167.

Liszkowski, Ulf, Malinda Carpenter, Anne Henning, Tricia Striano, \& Michael Tomasello (2004). Twelve-month-olds point to share attention and interest. Developmental Science, 7, 297-307.

Liszkowski, Ulf, Malinda Carpenter, Tricia Striano, \& Michael Tomasello (2006). Twelve- and 18-month-olds point to provide information for others. Journal of Cognition and Development, 7, 173-187.

Liszkowski, Ulf, Malinda Carpenter, \& Michael Tomasello (2007). Pointing out new news, old news, and absent referents at 12 months of age. Developmental Science, 10, 1-7.

Liszkowski, Ulf, Malinda Carpenter, \& Michael Tomasello (2008). Twelve-month-olds communicate helpfully and appropriately for knowledgeable and ignorant partners. Cognition, 108, 732-739.

Liszkowski, Ulf, Marie Schäfer, Malinda Carpenter, \& Michael Tomasello (2009). Prelinguistic infants, but not chimpanzees, communicate about absent entities. Psychological Science, 20, 654-660.

Lock, Andrew J., Andrew W. Young, Valerie Service, \& Penelope Chandler (1990). Some observations on the origins of the pointing gesture. In Virginia Volterra \& Carol. J. Erting (Eds.), From gesture to language in hearing and deaf children (pp. 42-55). Berlin: Springer-Verlag.

Masataka, Nobuo (2003). From index-finger extension to index-finger pointing: Ontogenesis of pointing in preverbal infants. In Sotaro Kita (Ed.), Pointing: where language, culture, and cognition meet, (pp.69-84). Mahwah, NJ: Lawrence Erlbaum Associates.

Masur, Elise F. (1983). Gestural development, dual-directional signaling, and the transition to words. Journal of Psycholinguistic Research, 12, 93-109.

Moore, Chris \& Valérie Corkum (1994). Social understanding at the end of the first year of life. Developmental Review, 14, 349-372.

Murphy, Catherine M. (1978). Pointing in a context of a shared activity. Child Development, 49, 371-380.

Pika, Simone (2008). Gestures of apes and pre-linguistic human children: Similar or different? First Language, 28, 116-140.

Pizzuto, Elena \& Micaela Capobianco (2005). The link and differences between deixis and symbols in children's early gestural-vocal system. Gesture, 5 (1), 179-199. 
Rowe, Meredith L. \& Susan Goldin-Meadow (2009). Early gesture selectively predicts later language learning. Developmental Science, 12, 182-187.

Rowe, Meredith L., Şeyda Özçaliskan, \& Susan Goldin-Meadow (2008). Learning words by hand: Gesture's role in predicting vocabulary development. First Language, 28, 182-199.

Saucier, Deborah M. \& Lorin J. Elias (2001). Lateral and sex differences in manual gesture during conversation. Laterality, 6, 239-245.

Tomasello, Michael (2006). Why don't apes point. In Nick J. Enfield, \& Stephen C. Levinson (Eds.), Roots of human sociality: culture, cognition and interaction (pp. 153-178). New York: Berg.

Tomasello, Michael, \& Joseph Call (1997). Primate cognition. New York: Oxford University Press.

Tomasello, Michael, Malinda Carpenter, \& Ulf Liszkowski (2007). A new look at infant pointing. Child Development, 78, 705-722.

Vauclair, Jacques \& Hélène Cochet (2010). Laterality for pointing and language development in toddlers. Manuscript submitted for publication.

Vauclair, Jacques \& Juliette Imbault (2009). Relationships between manual preferences for manipulative actions and for pointing gestures in infants and toddlers. Developmental Science, 12, 1060-1069.

Volterra, Virginia, Maria Cristina Caselli, Olga Capirci, \& Elena Pizzuto (2005). Gesture and the emergence and development of language. In Michael Tomasello \& Dan Slobin (Eds.), Beyond nature-nurture: essays in honor of Elizabeth Bates (pp. 3-30). Mahwah, NJ: Lawrence Erlbaum Associates.

Vygotsky, Lev S. (1988). Development of the higher mental functions. In Ken Richardson \& Sue Sheldon (Eds.), Cognitive development in adolescence (pp. 61-80). Hove: Lawrence Erlbaum.

Wilkins, David (2003). Why pointing with the index finger is not a universal (in sociocultural and semiotic terms). In Sotaro Kita (Ed.), Pointing: where language, culture, and cognition meet (pp. 171-215). Mahwah, NJ: Lawrence Erlbaum Associates.

Young, Andrew W., Andrew J. Lock, \& Valerie Service (1985). Infants' hand preferences for actions and gestures. Developmental Neuropsychology, 1, 17-27.

\section{Authors' addresses}

Hélène Cochet \& Jacques Vauclair

Center for Research in the Psychology of Cognition, Language \& Emotion

Department of Psychology

University of Provence

29, av. Robert Schuman

13621 Aix-en-Provence Cedex 1- France

helene.cochet@univ-provence.fr, Jacques.Vauclair@univ-provence.fr

\section{About the authors}

Hélène Cochet is a doctoral student who is conducting observational and experimental studies of the development of communicative abilities in infants and toddlers (see http://sites.univprovence.fr/wpsycle/membres/allocataires/cochet.html). 
Jacques Vauclair (PhD) is Professor of Developmental Psychology in the Department of Psychology (University of Provence, Aix-en-Provence, France) and senior member of the Institut Universitaire de France. He is the founder and director of the Center for Research in the Psychology of Cognition, Language, \& Emotion, in the Department of Psychology. His present research interests lie in the comparative study of gestural communication (CV and publications: http://sites.univ-provence.fr/wpsycle/membres/enseignants/vauclair.html). 
(C) 2010. John Benjamins Publishing Company All rights reserved 